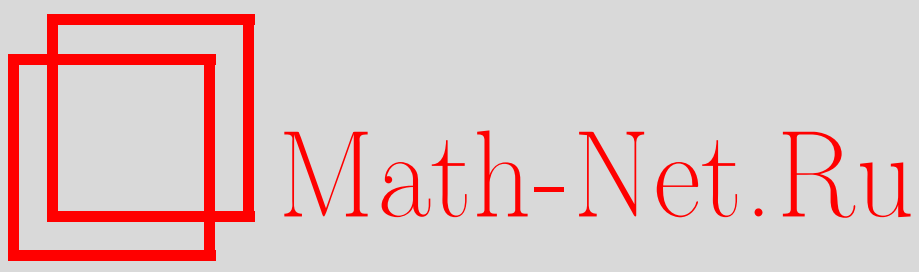

Э. Б. Винберг, Группы, задаваемые периодическими попарными соотношениями, матем. сб., 1997, том 188, номер 9, 3-12

DOI: https://doi.org/10.4213/sm254

Использование Общероссийского математического портала Math-Net.Ru подразумевает, что вы прочитали и согласны с пользовательским соглашением

http://www . mathnet.ru/rus/agreement

Параметры загрузки:

IP: 18.209 .158 .208

26 апреля 2023 г., 15:41:04 
УДК 512.04

\author{
Э. Б. Винберг
}

\title{
Группы, задаваемые периодическими попарными соотношениями
}

\begin{abstract}
Класс групп, задаваемьй периодическими попарньми соотношениями, включает в себя группы Кокстера и обобщенные треугольные группы. Для этого класса в статье доказываются обобщения некоторых теорем, известных для групп Кокстера и обобщенных треугольных групп.

Библиография: 16 названий.
\end{abstract}

Пусть $\Gamma$ - группа, порожденная элементами $x_{1}, \ldots, x_{n}$ с определяющими соотношениями

$$
w_{i}\left(x_{1}, \ldots, x_{n}\right)^{k_{i}}=e \quad(i=1, \ldots, m),
$$

где $w_{1}, \ldots, w_{m}-$ некоторые слова от $n$ букв. Говорят, что группа Г не сәсимается (в англоязычной литературе - does not collapse), если

$$
\operatorname{ord} w_{i}\left(x_{1}, \ldots, x_{n}\right)=k_{i}
$$

для любого $i$.

Гомоморфизм $\varphi: \Gamma \rightarrow G$ назьвается специальным, если

$$
\operatorname{ord} w_{i}\left(\varphi\left(x_{1}\right), \ldots, \varphi\left(x_{n}\right)\right)=k_{i}
$$

для любого $i$. Очевидно, что если группа Г допускает специальный гомоморфизм в какую-либо группу, то она не сжимается.

По лемме Сельберга [1] всякая конечнопорожденная линейная группа над полем нулевой характеристики обладает подгруппой конечного индекса без кручения. Поэтому если группа Г допускает специальное линейное представление (т.е. специальный гомоморфизм в группу $\left.\mathrm{GL}_{n}(\mathbb{C})\right)$, то она допускает специальньй гомоморфизм в конечную группу.

ТЕОРема 1. Пусть группа Г допускает специальный гомоморфизм в конечную группу. Если $\frac{1}{k_{1}}+\cdots+\frac{1}{k_{m}} \leqslant n-1$, то в Г имеется подгруппа конечного индекса, гомоморфно отображсающаяся на бесконечную ииклическую группу; если же $\frac{1}{k_{1}}+\cdots+\frac{1}{k_{m}}<n-1$, то в Г имеется подгруппа конечного индекса, гомоморфно отобрахсающаяся на свободную группу ранга 2.

Заметим, что если группа Г конечна и не сжимается, то ее тождественное отображение в себя является специальным гомоморфизмом в конечную групп. Поэтому из сформулированной теоремы вытекает

(C) Э.Б. Виньерг 1997 
СлЕДСТВИЕ. Если әруппа Г не сжимается $u \frac{1}{k_{1}}+\cdots+\frac{1}{k_{m}} \leqslant n-1$, mо Г бесконечна.

Последнее утверждение, насколько мне известно, было впервые доказано в обшем виде в работе Томаса [2]. В специальном случае обобщенных треугольных групп приведенная вьше теорема была доказана в работе Баумслага, Моргана и Шейлена [3]. Ее доказательство в общем виде будет дано в п. 1 настоящей работы.

Применение теоремы 1 упирается в построение специальных гомоморфизмов. Кроме того, такие гомоморфизмы могут дать важную информацию о группе Г и безотносительно к этой теореме, тем более что ее условия отнюдь не являются необходимыми для бесконечности группы.

Классическими примерами групп, для которых известно сушествование специальных (и к тому же точных) линейных представлений, являются треугольные гpynnbl

$$
T(k, l, m)=\left\langle x, y \mid x^{k}=y^{l}=(x y)^{m}=e\right\rangle \quad(k, l, m \geqslant 2)
$$

и группь Кокстера

$$
\left\langle x_{1}, \ldots, x_{n} \mid x_{i}^{2}=e(1 \leqslant i \leqslant n),\left(x_{i} x_{j}\right)^{k_{i j}}=e(1 \leqslant i<j \leqslant n)\right\rangle \quad\left(k_{i j} \geqslant 2\right) .
$$

В этих примерах можно допустить, что некоторые показатели равны $\infty$, что означает просто отсутствие соответствуюшего соотношения.

Геометрическое представление треугольных групп как дискретных групп движений сферы, евклидовой плоскости или плоскости Лобачевского и, тем самьм, их точное линейное представление было получено Диком и Пуанкаре в конце прошлого века. Специальное линейное представление любой группы Кокстера было построено самим Кокстером [4] в 1935 г. Как доказал Титс [5], это представление является точным. Отметим, что теория треугольных групп вкладывается в теорию групп Кокстера, так как треугольные группы - это не что иное, как четные (т.е. образованные словами четной длины) подгруппы в группах Кокстера с тремя образующими.

В случае $k_{i j}=\infty$ элемент $x_{i} x_{j}$ групшы Кокстера при упомянутом выше линейном представлении переходит в унипотентный оператор, отличный от единицы.

Треугольная группа $T(k, l, m)$ конечна тогда и только тогда, когда она реализуется на сфере, что имеет место в точности тогда, когда $\frac{1}{k}+\frac{1}{l}+\frac{1}{m}>1$. Таким образом, в случае треугольных групп условие следствия теоремы 1 не только достаточно, но и необходимо для бесконечности группы. В случае групп Кокстера это уже не так. Все конечные группы Кокстера найдены в той же работе [4]. В настояшее время они широко известны благодаря тому, что, за немногими исключениями, они являются группами Вейля полупростых групп Ли.

В работах Файна и Розенбергера [6] и Баумслага, Моргана и Шейлена [3] были рассмотрены обобщенные треугольные группь

$$
\left\langle x, y \mid x^{k}=y^{l}=w(x, y)^{m}=e\right\rangle,
$$

где $k, l, m \geqslant 2$, а $w$ - циклически приведенное слово, не сводящееся к степени $x$ или $y$ с учетом соотношений $x^{k}=y^{l}=e$; можно считать, что

$$
w(x, y)=x^{k_{1}} y^{l_{1}} \cdots x^{k_{s}} y^{l_{s}} \quad\left(0<k_{i}<k, 0<l_{i}<l\right) .
$$


В работе [3] было доказано, что всякая обобщенная треугольная группа допускает специальный гомоморфизм в группу $\mathrm{PSL}_{2}(\mathbb{C})$. (Другое доказательство и обобщение этого результата см. в [7].) В п. 2 настоящей работы будет приведен удобный для нас вариант доказательства этой теоремы.

Все конечные обобшенные треугольные группы, за исключением двух случаев, остававшихся неясными, были классифицированы в работе [8]. Оставшиеся два случая были разобраны в [9].

В настоящей работе рассматривается класс групп, включающий в себя как группы Кокстера, так и обобщенные треугольные группы. Это группы вида

$$
\left\langle x_{1}, \ldots, x_{n} \mid x_{i}^{k_{i}}=e(1 \leqslant i \leqslant n), w_{i j}\left(x_{i}, x_{j}\right)^{k_{i j}}=e(1 \leqslant i<j \leqslant n)\right\rangle,
$$

где $k_{i}, k_{i j} \geqslant 2$, а $w_{i j}$ - любые слова, удовлетворяющие тому же условию, что и в определении обобшенных треугольных групп. Они назьваются в работе групnами, задаваемыми периодическими попарными соотночениями.

Основным результатом работы является

ТЕОРема 2. Всякая группа с п образующими, задаваемая периодическими попарными соотношениями, допускает специальный гомоморфизм в груп$n y \mathrm{SO}_{n+1}(\mathbb{C})$.

Так как $\mathrm{SO}_{3}(\mathbb{C}) \simeq \mathrm{PSL}_{2}(\mathbb{C})$, то эта теорема является прямым обобщением теоремы Баумслага, Моргана и Шейлена.

Доказательство теоремы 2 комбинирует идеи работы [3] и работы Царанова [10], в которой эта теорема была доказана для случая

$$
w_{i j}=x_{i}^{p_{i j}} x_{j}^{q_{i j}}, \quad\left(p_{i j}, k_{i}\right)=\left(q_{i j}, k_{j}\right)=1 .
$$

В работе Царанова [11] были классифицированы все конечные групшы, задаваемые периодическими попарными соотношениями такого вида.

По аналогии с треугольньми группами естественно называть тетраэдральньми четные подгруппы групп Кокстера с четырьмя образуюшими. Они имеют копредставление вида

$$
\left\langle x, y, z \mid x^{k}=y^{l}=z^{m}=\left(y z^{-1}\right)^{p}=\left(z x^{-1}\right)^{q}=\left(x y^{-1}\right)^{r}=e\right\rangle .
$$

Соответственно этому группы с тремя образуюшими, задаваемые периодическими попарньми соотношениями, естественно называть обобщенными тетраәдральнъци группами. Для них теорема 2 может быть усилена.

ТЕОРЕМА 3. Всякая обобщенная тетраэдральная группа допускает специальньй гомоморфизм в группу $\mathrm{SO}_{3}(\mathbb{C})\left(\simeq \mathrm{PSL}_{2}(\mathbb{C})\right)$.

Доказательство этой теоремы, как мы увидим, может быть получено незначительной модификацией доказательства теоремы 2 . Ее независимое доказательство, основанное на теории инвариантов (также приводимое ниже), было получено мной еше в 1991 г. и докладывалось в том же году в Москве, Бохуме, Билефельде и Базеле, но не было опубликовано до сих пор.

Я благодарю $\Phi$. Левина, Г. Розенбергера и М. Хагельберга за полезные обсуждения. 
1. Доказательство теоремы 1. Пусть $\Gamma_{0} \subset \Gamma$ - ядро гомоморфизма, о котором идет речь в теореме. Из условия следует, что $\left|\Gamma: \Gamma_{0}\right|=d<\infty$ и $\Gamma_{0}$ не содержит элементов вида $w_{i}\left(x_{1}, \ldots, x_{n}\right)^{k}, 0<k<k_{i}$.

Пусть $F$ - свободная группа с образующими $u_{1}, \ldots, u_{n}$ и $\pi: F \rightarrow \Gamma$ - гомоморфизм, отображающий $u_{i}$ в $x_{i}$. Положим $F_{0}=\pi^{-1}\left(\Gamma_{0}\right)$. Это (нормальная) подгруппа индекса $d$ в $F$ и, следовательно, свободная группа ранга $d(n-1)+1$.

Ядро $N$ гомоморфизма $\pi$ как нормальная подгруппа группы $F_{0}$ порождается элементами вида

$$
v w_{i}\left(u_{1}, \ldots, u_{n}\right)^{k_{i}} v^{-1}
$$

где $v$ пробегает представителей смежных классов $F$ по $F_{0}$. Более того, ясно, что для каждого $i$ на самом деле достаточно взять представителей смежных классов $F$ по произведению $F_{0}$ и циклической подгрупшы, порожденной элементом $w_{i}\left(u_{1}, \ldots, u_{n}\right)$. Так как $F_{0}$ не содержит элементов вида $w_{i}\left(u_{1}, \ldots, u_{n}\right)^{k}, 0<k<k_{i}$, то имеется $d / k_{i}$ таких представителей. Следовательно, группа $N$ порождена $d \sum_{i} \frac{1}{k_{i}}$ элементами как нормальная подгруппа групшы $F_{0}$.

Таким образом, группа $\Gamma_{0}$ имеет $d(n-1)+1$ образующих и $d \sum_{i} \frac{1}{k_{i}}$ определяющих соотношений. Если $\sum_{i} \frac{1}{k_{i}} \leqslant n-1$, то число определяюших соотношений групшы $\Gamma_{0}$ меньше числа образующих и, следовательно, она гомоморфно отображается на свободную циклическую группу. Если же $\sum_{i} \frac{1}{k_{i}}<n-1$, то число определяющих соотношений по меньшей мере на два менше числа образующих и, следовательно [12], в Г и имееся подгруппа конечного индекса, гомоморфно отображающаяся на свободную группу ранга 2.

2. Доказательство теоремы 2 при $n=3$. Пусть $V$-комплексное векторное пространство с базисом $\left\{e_{0}, e_{1}, e_{2}\right\}$ и скалярньм умножением (быть может, вырожденным), определенньп на базисных векторах формулами

$$
\begin{aligned}
& \left(e_{0}, e_{0}\right)=\left(e_{1}, e_{1}\right)=\left(e_{2}, e_{2}\right)=1, \\
& \left(e_{0}, e_{1}\right)=\left(e_{0}, e_{2}\right)=0, \\
& \left(e_{1}, e_{2}\right)=z \in \mathbb{C} .
\end{aligned}
$$

Рассмотрим ортогональньй оператор $X_{1}$, представляющий собой поворот на угол $2 \pi / k_{1}$ в плоскости $\left\langle e_{0}, e_{1}\right\rangle$ и тождественно действующий в ортогональном дополнении. Легко видеть, что в базисе $\left\{e_{0}, e_{1}, e_{2}\right\}$ он задается матрицей

$$
\left(\begin{array}{ccc}
\cos \frac{2 \pi}{k_{1}} & -\sin \frac{2 \pi}{k_{1}} & -z \sin \frac{2 \pi}{k_{1}} \\
\sin \frac{2 \pi}{k_{1}} & \cos \frac{2 \pi}{k_{1}} & z\left(\cos \frac{2 \pi}{k_{1}}-1\right) \\
0 & 0 & 1
\end{array}\right) .
$$

Аналогично, ортогональный оператор $X_{2}$, представляющий собой поворот на угол $2 \pi / k_{2}$ в плоскости $\left\langle e_{0}, e_{2}\right\rangle$, задается матрицей

$$
\left(\begin{array}{ccc}
\cos \frac{2 \pi}{k_{2}} & -z \sin \frac{2 \pi}{k_{2}} & -\sin \frac{2 \pi}{k_{2}} \\
0 & 1 & 0 \\
\sin \frac{2 \pi}{k_{2}} & z\left(\cos \frac{2 \pi}{k_{2}}-1\right) & \cos \frac{2 \pi}{k_{2}}
\end{array}\right) .
$$


Пользуясь тем, что число $z$ находится в нашем распоряжении, постараемся выбрать его так, чтобы (ортогональньй) оператор $w_{12}\left(X_{1}, X_{2}\right)$ был поворотом на угол $2 \pi / k_{12}$ в некоторой невырожденной плоскости. Для этого необходимо и достаточно, чтобы след этого оператора был равен $2 \cos \frac{2 \pi}{k_{12}}+1$. (Отметим, что если скалярное умножение в $V$ вырожденно, то все рассматриваемые операторы тождественно действуют на его ядре.)

Можно считать, что

$$
w_{12}\left(x_{1}, x_{2}\right)=x_{1}^{p_{11}} x_{2}^{p_{21}} \cdots x_{1}^{p_{1 s}} x_{2}^{p_{2 s}} \quad\left(0<p_{1 i}<k_{1}, 0<p_{2 i}<k_{2}\right)
$$

тогда след оператора $w_{12}\left(X_{1}, X_{2}\right)$ есть многочлен степени $\leqslant 2 s$ от $z$, коэффициент которого при $z^{2 s}$ равен

$$
\prod_{i}\left(\cos \frac{2 \pi p_{1 i}}{k_{1}}-1\right)\left(\cos \frac{2 \pi p_{2 i}}{k_{2}}-1\right) \neq 0
$$

Таким образом, это многочлен положительной степени. Следовательно, он принимает все значения, в том числе и интересующее нас.

Если число $z$ выбрано указанным образом, то отображение $x_{1} \mapsto X_{1}, x_{2} \mapsto X_{2}$ продолжается до специального гомоморфизма группы $Г$ в группу ортогональных преобразований пространства $V$. Тем самьм, если скалярное умножение в $V$ невырожденно, мы получим специальный гомоморфизм группы $\Gamma$ в $\mathrm{SO}_{3}(\mathbb{C})$. Если оно вырожденно, то, перейдя к факторпространству по его ядру, мы получим специальный гомоморфизм группы $\Gamma$ в $\mathrm{SO}_{2}(\mathbb{C}) ;$ но так как $\mathrm{SO}_{2}(\mathbb{C}) \subset \mathrm{SO}_{3}(\mathbb{C})$, то и в этом случае сушествует специальный гомоморфизм группы $\Gamma$ в $\mathrm{SO}_{3}(\mathbb{C})$.

3. Доказательство теоремы 2 в общем случае. Пусть $V$ - комплексное векторное пространство с базисом $\left\{e_{0}, e_{1}, \ldots, e_{n}\right\}$ и скалярньм умножением, определенным на базисных векторах формулами

$$
\begin{aligned}
& \left(e_{0}, e_{0}\right)=\left(e_{1}, e_{1}\right)=\cdots=\left(e_{n}, e_{n}\right)=1 \\
& \left(e_{0}, e_{1}\right)=\cdots=\left(e_{0}, e_{n}\right)=0 \\
& \left(e_{i}, e_{j}\right)=z_{i j} \in \mathbb{C} \quad(1 \leqslant i<j \leqslant n)
\end{aligned}
$$

Обозначим через $X_{i}(i=1, \ldots, n)$ ортогональный оператор, представляющий собой поворот на угол $2 \pi / k_{i}$ в плоскости $\left\langle e_{0}, e_{i}\right\rangle$ и тождественно действующий в ортогональном дополнении.

В соответствии с п. 2 подберем числа $z_{i j}$ таким образом, чтобы оператор $w_{i j}\left(X_{i}, X_{j}\right)$ действовал в пространстве $\left\langle e_{0}, e_{i}, e_{j}\right\rangle$ как поворот на угол $2 \pi / k_{i j}$ в некоторой невырожденной плоскости. Докажем, что в ортогональном дополнении к этой плоскости (во всем пространстве $V$, а не только в $\left.\left\langle e_{0}, e_{i}, e_{j}\right\rangle\right)$ этот оператор будет действовать тождественно. Это очевидно, если подпространство $\left\langle e_{0}, e_{i}, e_{j}\right\rangle$ невырожденно (т.е. если $z_{i j} \neq \pm 1$ ): действительно, в этом случае оба оператора $X_{i}, X_{j}$, а следовательно, и оператор $w_{i j}\left(X_{i}, X_{j}\right)$, действуют тождественно в ортогональном дополнении к $\left\langle e_{0}, e_{i}, e_{j}\right\rangle$. Таким образом, при $z_{i j} \neq \pm 1$ размерность пространства неподвижных точек оператора $w_{i j}\left(X_{i}, X_{j}\right)$ равна $n-1$. 
Путем предельного перехода отсюда получаем, что это верно и при $z_{i j}= \pm 1$. Это и означает, что оператор $w_{i j}\left(X_{i}, X_{j}\right)$ действует тождественно в ортогональном дополнении к плоскости поворота.

Ясно, что отображение $x_{i} \mapsto X_{i}$ продолжается до специального гомоморфизма группы $Г$ в группу ортогональных преобразований пространства $V$. Если скалярное умножение в $V$ невырожденно, то мы тем самым получаем специальный гомоморфизм группы $\Gamma$ в группу $\mathrm{SO}_{n+1}(\mathbb{C})$. Если оно вырожденно, то все рассматриваемые операторы тождественно действуют на его ядре. Перейдя к факторпространству, мы получим специальный гомоморфизм группы $\Gamma$ в $\mathrm{SO}_{m}(\mathbb{C}), m \leqslant n$; но так как $\mathrm{SO}_{m}(\mathbb{C}) \subset \mathrm{SO}_{n+1}(\mathbb{C})$, то и в этом случае сушествует специальньй гомоморфизм групшы $\Gamma$ в $\mathrm{SO}_{n+1}(\mathbb{C})$.

4. Доказательство теоремы 3. В случае $n=3$ мы построили специальный гомоморфизм группы $Г$ в группу $\mathrm{SO}_{4}(\mathbb{C})$. Известно, что

$$
\mathrm{SO}_{4}(\mathbb{C}) \simeq\left(\mathrm{SL}_{2}(\mathbb{C}) \times \mathrm{SL}_{2}(\mathbb{C})\right) /\{(E, E),(-E,-E)\}
$$

и, следовательно, в $\mathrm{SO}_{4}(\mathbb{C})$ имеется нормальная подгруппа $H$, изоморфная $\mathrm{SL}_{2}(\mathbb{C})$, факторгруппа по которой изоморфна $\mathrm{PSL}_{2}(\mathbb{C})\left(\simeq \mathrm{SO}_{3}(\mathbb{C})\right)$. Переходя к этой факторгруппе, мы получаем гомоморфизм группы $\Gamma$ в $\mathrm{PSL}_{2}(\mathbb{C})$. Докажем, что он специален. Для этого нужно только проверить, что подгруппа $H$ не содержит нетривиальных степеней операторов $X_{i}$ и $w_{i j}\left(X_{i}, X_{j}\right)$.

Заметим, что согласно построению каждый из указанных операторов, а также любая его нетривиальная степень, полупрост и имеет двукратное собственное значение 1 . С другой стороны, спектр любого оператора из $H$ имеет вид $\left\{\lambda, \lambda, \lambda^{-1}, \lambda^{-1}\right\}$. Это и доказывает сформулированное выше утверждение.

5. Инварианты системы матриц. Рассмотрим действие группы $\mathrm{SL}_{2}(\mathbb{C})$ на пространстве $M_{2}(\mathbb{C})$ всех матриц сопряжениями. Определитель матрицы есть невырожденная квадратичная форма на $M_{2}(\mathbb{C})$, инвариантная относительно этого действия. Введем в $M_{2}(\mathbb{C})$ инвариантное скалярное умножение так, чтобы

$$
(X, X)=-2 \operatorname{det} X \text {. }
$$

Пространство $M_{2}(\mathbb{C})$ разлагается в ортогональную прямую сумму одномерного пространства $\langle E\rangle$, на котором $\mathrm{SL}_{2}(\mathbb{C})$ действует тривиально, и трехмерного подпространства $M_{2}^{0}(\mathbb{C})$ матриц с нулевым следом, на котором $\mathrm{SL}_{2}(\mathbb{C})$ действует как группа $\mathrm{SO}_{3}(\mathbb{C})$ всех унимодулярных ортогональных преобразований. Отметим, что проекция матрицы $X$ на подпространство $\langle E\rangle$ равна $\frac{1}{2}(\operatorname{tr} X) E$.

Имея в виду дальнейшие приложения, приведем здесь описание инвариантов системы матриц $X_{1}, \ldots, X_{m} \in M_{2}(\mathbb{C})$ относительно действия группы $\mathrm{SL}_{2}(\mathbb{C})$ одновременными сопряжениями. Ввиду сказанного выше таковыми инвариантами являются следы матриц $X_{1}, \ldots, X_{m}$ плюс инварианты системы их проекций на $M_{2}^{0}(\mathbb{C})$ относительно действия группы $\mathrm{SO}_{3}(\mathbb{C})$.

Описание образуюших и определяюших соотношений алгебры инвариантов системы векторов $x_{1}, \ldots, x_{m} \in \mathbb{C}^{n}$ относительно действия группы $\mathrm{SO}_{n}(\mathbb{C})$ есть один из основных результатов классической теории инвариантов XIX века (см., например, [13]). В частности, известно, что при $m<n$ алгебра инвариантов свободно 
порождается скалярньми произведениями векторов $x_{1}, \ldots, x_{m}$. При $m=n$ есть еше инвариант $\operatorname{det}\left(x_{1}, \ldots, x_{n}\right)$, равный определителю матрицы, составленной из координат векторов $x_{1}, \ldots, x_{n}$ в каком-либо фиксированном базисе. Его квадрат выражается через скалярные произведения векторов $x_{1}, \ldots, x_{n}$, а именно, равен определителю Грама этих векторов; других соотношений между образующими инвариантами в этом случае нет. Случай $m>n$ более сложен, но он нам не понадобится.

Если матрица $X \in M_{2}^{0}(\mathbb{C})$ имеет собственные значения $\lambda,-\lambda$, то

$$
\operatorname{tr} X^{2}=2 \lambda^{2}=-2 \operatorname{det} X=(X, X) .
$$

Поэтому скалярное умножение на $M_{2}^{0}(\mathbb{C})$ может быть задано формулой

$$
(X, Y)=\operatorname{tr} X Y
$$

Далее, легко видеть, что

$$
X Y+Y X=(X, Y) E .
$$

Отсюда следует, что $\operatorname{tr} X Y Z$ есть кососимметрическая трилинейная форма на $M_{2}^{0}(\mathbb{C})$. Поэтому можно считать, что

$$
\operatorname{det}(X, Y, Z)=\operatorname{tr} X Y Z
$$

Для любых матриц $X, Y \in M_{2}(\mathbb{C})$ скалярное произведение их проекций на $M_{2}^{0}(\mathbb{C})$ равно

$$
\operatorname{tr}\left(X-\frac{1}{2}(\operatorname{tr} X) E\right)\left(Y-\frac{1}{2}(\operatorname{tr} Y) E\right)=\operatorname{tr} X Y-\frac{1}{2}(\operatorname{tr} X)(\operatorname{tr} Y)
$$

Аналогично, для матриц $X, Y, Z \in M_{2}(\mathbb{C})$ определитель их проекций на $M_{2}^{0}(\mathbb{C})$ есть $\operatorname{tr} X Y Z$ плюс некоторый многочлен от $\operatorname{tr} Y Z, \operatorname{tr} Z X, \operatorname{tr} X Y, \operatorname{tr} X, \operatorname{tr} Y, \operatorname{tr} Z$ (линейный по каждой из матриц $X, Y, Z$ ).

Из всего сказанного следует, что алгебра инвариантов пары матриц $X, Y \in$ $M_{2}(\mathbb{C})$ относительно действия группы $\mathrm{SL}_{2}(\mathbb{C})$ свободно порождается многочленами $\operatorname{tr} X, \operatorname{tr} Y, \operatorname{tr} X^{2}, \operatorname{tr} Y^{2}, \operatorname{tr} X Y$, а алгебра инвариантов тройки матриц $X, Y, Z \in$ $M_{2}(\mathbb{C})$ порождается многочленами $\operatorname{tr} X, \operatorname{tr} Y, \operatorname{tr} Z, \operatorname{tr} X^{2}, \operatorname{tr} Y^{2}, \operatorname{tr} Z^{2}, \operatorname{tr} Y Z, \operatorname{tr} Z X$, $\operatorname{tr} X Y, \operatorname{tr} X Y Z$ с единственным определяющим соотношением вида

$$
(\operatorname{tr} X Y Z)^{2}+P \cdot \operatorname{tr} X Y Z+Q=0
$$

где $P$ и $Q$ - какие-то многочлены от первых девяти образующих.

Ввиду соотношения

$$
\operatorname{tr} X^{2}=(\operatorname{tr} X)^{2}-2 \operatorname{det} X
$$

вьполняющегося для любой матрицы $X \in M_{2}(\mathbb{C})$, все следы квадратов в приведенных выше системах образующих алгебр инвариантов можно заменить определителями. Если же нас интересуют инварианты систем унимодулярных матриц, 
то их вообще можно выкинуть. Таким образом, мы получили, что алгебра инвариантов пары матрии $X, Y \in \mathrm{SL}_{2}(\mathbb{C})$ относительно действия группь $\mathrm{SL}_{2}(\mathbb{C})$ одновременными сопрязсениями свободно порожсдается многочленами

$\operatorname{tr} X, \operatorname{tr} Y, \operatorname{tr} X Y$

а алгебра инвариантов тройки матрич $X, Y, Z \in \mathrm{SL}_{2}(\mathbb{C})$ порожсдается многочленами

$\operatorname{tr} X, \operatorname{tr} Y, \operatorname{tr} Z, \operatorname{tr} Y Z, \operatorname{tr} Z X, \operatorname{tr} X Y, \operatorname{tr} X Y Z$

с единственным определяющим соотношением вида

$$
(\operatorname{tr} X Y Z)^{2}+p \cdot \operatorname{tr} X Y Z+q=0
$$

где р и q-какие-то многочлены от первых шести образующих.

Отметим, что эти утверждения отнюдь не являются новьми, хотя трудно сказать, кем и когда они были впервые доказаны. Их можно, конечно, извлечь из соответствующих утверждений для матриц любого порядка, доказанных в [14] и [15], но несомненно, что они были известны ранњше.

Применение этих утверждений в настоящей статье основано на следующей теореме теории инвариантов (см., например, [16]): для всякой редуктивной комплексной алгебраической группь $G$, действующей на аффинном алгебрачческом многообразии $X$, морфизм факторизачии

$$
\pi: X \rightarrow X / / G \doteqdot \operatorname{Spec} \mathbb{C}[X]^{G}
$$

сюргективен.

Здесь $\mathbb{C}[X]^{G}$ обозначает алгебру $G$-инвариантных многочленов на $X$. Морфизм $\pi$ определяется ее тождественньм вложением в алгебру $\mathbb{C}[X]$ всех многочленов на $X$. Если алгебра $\mathbb{C}[X]^{G}$ порождается многочленами $f_{1}, \ldots, f_{m}$, то многообразие $X / / G$ может быть реализовано в $\mathbb{C}^{m}$ как множество нулей идеала $I$ алгебраических соотношений между $f_{1}, \ldots, f_{m}$; морфизм $\pi$ при этом задается формулой

$$
\pi(x)=\left(f_{1}(x), \ldots, f_{m}(x)\right)
$$

Сформулированная выше теорема означает, что для любых чисел $c_{1}, \ldots, c_{m}$, удовлетворяюших всем алгебраическим соотношениям, которьм удовлетворяют многочлены $f_{1}, \ldots, f_{m}$, сушествует такая точка $x \in X$, что

$$
f_{i}(x)=c_{i} \quad(i=1, \ldots, m)
$$

(Отметим, что для любых многочленов $f_{1}, \ldots, f_{m}$ это, вообше говоря, неверно.) 
6. Теоретико-инвариантное доказательство теоремы 3. Для любого натурального $m$ имеем

$$
\frac{z^{m}-z^{-m}}{z-z^{-1}}=P_{m}\left(z+z^{-1}\right)
$$

где $P_{m}$ - многочлен степени $m-1$ (многочлен Чебышёва второго рода с точностью до линейной замены переменной), имеющий нули в точках

$$
2 \cos \frac{\pi}{m}, 2 \cos \frac{2 \pi}{m}, \ldots, 2 \cos \frac{(m-1) \pi}{m} .
$$

Путем несложных преобразований получаем

$$
z^{m}=P_{m}\left(z+z^{-1}\right) z-P_{m-1}\left(z+z^{-1}\right) .
$$

Следовательно,

$$
Z^{m}=P_{m}(\operatorname{tr} Z) Z-P_{m-1}(\operatorname{tr} Z) E
$$

для любой матрицы $Z \in \mathrm{SL}_{2}(\mathbb{C})$.

Пусть $w$ - слово вида (2). Для матриц $X, Y \in \mathrm{SL}_{2}(\mathbb{C})$, пользуясь соотношением (4), нетрудно представить $\operatorname{tr} w(X, Y)$ в виде многочлена $P_{w}$ степени $\leqslant s$ от $\operatorname{tr} X Y$, коэффициенты которого являются многочленами от $\operatorname{tr} X$ и $\operatorname{tr} Y$. При этом коэффициент при $(\operatorname{tr} X Y)^{s}$ будет равен

$$
\prod_{i=1}^{s} P_{k_{i}}(\operatorname{tr} X) P_{l_{i}}(\operatorname{tr} Y)
$$

Если $\operatorname{tr} X=2 \cos \frac{\pi}{k}$ и $\operatorname{tr} Y=2 \cos \frac{\pi}{l}$, то этот коэффициент не равен нулю и, следовательно, $P_{w}$ - многочлен положительной степени. (Отметим, что этот результат приведен в [3] со ссылкой на Т. Йоргенсена.) В частности, для любого $m$ сушествует такое число $\zeta_{m}$, что

$$
P_{w}\left(\zeta_{m}\right)=2 \cos \frac{\pi}{m} .
$$

С другой стороны, в силу результатов, сформулированных в предыдушем пункте, для любых $k, l, m$ сушествуют такие матрицы $X, Y \in \mathrm{SL}_{2}(\mathbb{C})$, что

$$
\operatorname{tr} X=2 \cos \frac{\pi}{k}, \operatorname{tr} Y=2 \cos \frac{\pi}{l}, \operatorname{tr} X Y=\zeta_{m} .
$$

При $k, l, m \geqslant 2$ порядки $X, Y$ и $w(X, Y)$ в $\mathrm{PSL}_{2}(\mathbb{C})$ равны тогда $k, l$ и $m$ соответственно, что дает нам специальный гомоморфизм обобщенной треугольной группы $(1)$ в $\mathrm{PSL}_{2}(\mathbb{C})$.

Для обобщенных тетраэдральных групп ситуация несколько осложняется тем, что алгебра инвариантов системы трех матриц не является свободной. Однако из вида соотношения (3) следует, что образуюшие

$$
\operatorname{tr} X, \operatorname{tr} Y, \operatorname{tr} Z, \operatorname{tr} Y Z, \operatorname{tr} Z X, \operatorname{tr} X Y
$$

могут принимать независимо друг от друга произвольные значения. После этого замечания построение специального гомоморфизма обобщенной тетраэдральной группы в $\mathrm{PSL}_{2}(\mathbb{C})$ проводится так же, как для обобшенных треугольных групп. 


\section{Список литературы}

1. Сельберг А. О дискретных группах преобразований симметрических пространств большой размерности // Математика. 1962. Т. 6. №3. С. 3-15.

2. Thomas R. M. Cayley graphs and group presentations // Math. Proc. Cambridge Phil. Soc. 1988. V. 103. № 3. P. 385-387.

3. Baumslag G., Morgan J. W., Shalen P. B. Generalized triangle groups // Math. Proc. Cambridge Phil. Soc. 1987. V. 102. №1. P. 25-31.

4. Coxeter H.S.M. The complete enumeration of finite groups of the form $R_{i}^{2}=$ $\left(R_{i} R_{j}\right)^{k_{i j}}=1 / /$ J. London Math. Soc. 1935. V. 10. P. 21-25.

5. Бурбаки Н. Группы и алгебры Ли. Гл. IV-VI. М.: Мир, 1972.

6. Fine B., Rosenberger G. A note on generalized triangle groups // Abh. Math. Sem. Univ. Hamburg. 1986. V. 56. P. 233-244.

7. Fine B., Howie J., Rosenberger G. One-relator quotients and free products of cyclics // Proc. Amer. Math. Soc. 1988. V. 102. № 2. P. 249-254.

8. Howie J., Metaftsis V., Thomas R. M. Finite generalized triangle groups // Trans. Amer. Math. Soc. 1995. V. 347. №9. P. 3613-3623.

9. Lévai L., Rosenberger G., Souvignier B. All finite generalized triangle groups // Trans. Amer. Math. Soc. (to appear).

10. Tsaranov S. V. On a generalization of Coxeter groups // Algebras, Groups and Geometries. 1989. V. 6. P. 281-318.

11. Tsaranov S. V. Finite generalized Coxeter groups // Algebras, Groups and Geometries. 1989. V. 6. P. 421-452.

12. Baumslag B., Pride S.J. Groups with two more generators than relators // J. London Math. Soc. 1978. V. 17. P. 425-426.

13. Вейль Г. Классические группы, их инварианты и представления. М.: ИЛ, 1947.

14. Размыслов Ю. П. Тождества со следом полных матричных алгебр над полем характеристики 0 // Изв. АН. СССР. Сер. матем. 1974. Т. 38. № 4. С. 723-756.

15. Procesi $C$. The invariant theory of $n \times n$ matrices // Adv. in Math. 1976. V. 19. P. 306-381.

16. Винберг Э.Б., Попов В.Л. Теория инвариантов // Итоги науки и техники. Совр. пробл. матем. Фундам. направл.. Т. 55. М.: ВИНИТИ, 1989. С. 137-309.

Московский государственный

университет им. М. В. Ломоносова
Поступила в редакцию 09.01 .1997 